\title{
Performance and Upgrade Plans for the CMS Hadron Calorimeter at the LHC
}

\author{
Sudarshan Paramesvaran* \\ On behalf of the CMS Collaboration \\ University of California, Riverside \\ E-mail: sudanecern.ch
}

The hadron calorimeters of the CMS experiment have successfully recorded data at $7 \mathrm{TeV}$ and 8 $\mathrm{TeV}$ center-of-mass energy during 2011 and $2012 \mathrm{LHC}$ operation. The performance of all systems (barrel, end-cap, forward and the outer calorimeters) are discussed and results from the full 2011 dataset are shown on noise rejection, calibration, collision timing, and identification of jet candidates and for other salient features. In addition, the CMS collaboration is planning improvements to the hadron calorimeters which include the replacement of the HPD photodetectors with SiPMs, increased depth segmentation in the calorimeter, and the inclusion of TDC capability. The status of the Research and Development for these upgrades will be discussed, including the testing of the upgraded microTCA readout electronics during current LHC data taking.

36th International Conference on High Energy Physics,

July 4-11, 2012

Melbourne, Australia

\footnotetext{
* Speaker.
} 


\section{Introduction}

The CMS detector at CERN [1] was constructed to record and study high energy collisions produced by the LHC. The Hadron Calorimeter (HCAL) plays a crucial role in helping to identify hadronic jets, and exotic particles through their missing transverse energy signatures. The CMS HCAL [2]is split into 4 parts, Hcal Barrel (HB), Hcal Endcaps (HE), Hcal Forward (HF), and Hcal Outer (HO). HB and HE are positioned after the tracker, but still within the 3.8T solenoidal field. The $\mathrm{HO}$ is present to measure late developing showers and to improve the energy resolution of particles depositing energy after HB. HB, HE and HO use plastic scintillator tiles interspersed between stainless steel and brass absorber plates. The signal readout occurs via Hybrid Photodiodes (HPD), which are designed to operate within a 4T magnetic field. The HF calorimeters are positioned $11.2 \mathrm{~m}$ away from the interaction point and extend the pseudorapidity coverage to $|\eta=5|$. They are made of steel absorbers, with embedded quartz fibers which generate cerenkov signals. Conventional photo-miltiplier tubes (PMT) are used to read out the signals. This note will consider the performance of the different HCAL calorimeters in the LHC 7 (2011) and $8 \mathrm{TeV}$ (2012) runs, and will outline various techniques used to monitor and control the stability of the HCAL. In addition the proposed upgrades to the hadron calorimeters will be discussed - including the testing of devices during current LHC data-taking.

The Technical Design Report of the Phase 1 upgrade of the CMS HCAL is listed at [3].

\section{HCAL Readout and Calibration}

The stability of the response of the HPD's and PMT's is monitored using a calibration system consisting of a UV laser and LED's. Dedicated calibration runs are recorded in between LHC fills, and in the orbit-gap between LHC bunch-trains. The data obtained using the laser is used primarily to measure the timing of the signals. Furthermore, collision data is used to obtain calorimeter response corrections.

\subsection{HPD Gain Shift}

The HPD's in HB and HE have been monitored since the start of LHC operation in 2010 until the present. Figure 1 (left) shows the drift of each HPD as a function of time, since April 2010. Most of the HPD's are stable within 2 to $3 \%$ but there are a few that exhibit a shift greater than $10 \%$. This is more clearly seen in Figure 1 (right), which shows the drift of each of the 5184 HPD's relative to the start of data-taking in 2010.

\subsection{HF PMT Response loss}

The PMTs in HF are using the LED calibration system discussed above. Figure 2 shows the loss in different $\eta$ regions of the HF. The loss is more pronounced in higher eta regions, which corresponds to regions which have a higher particle flux, thus drawing more current from the PMTs. One can see that the rate of loss levels out with increasing luminosity - this is due to the rate of current being drawn stabilising.

Corrections for HBHE gain drift and HF PMT response loss are made to calibration coefficients to ensure the effects are accounted for. 

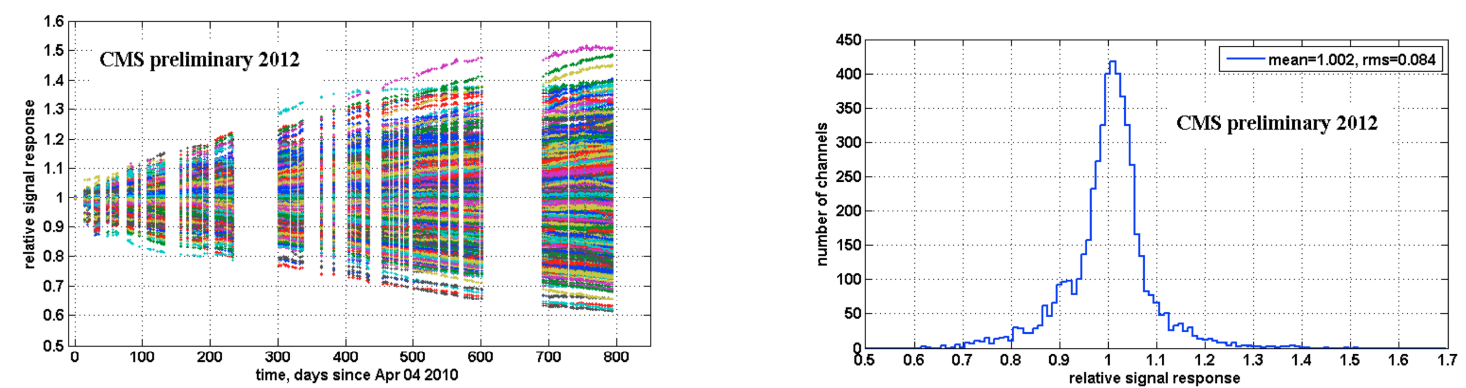

Figure 1: Response of HPD channels as a function of time, since the start of LHC operation in 2010.

R7525 Hamamatsu (Single-anode PMTs)

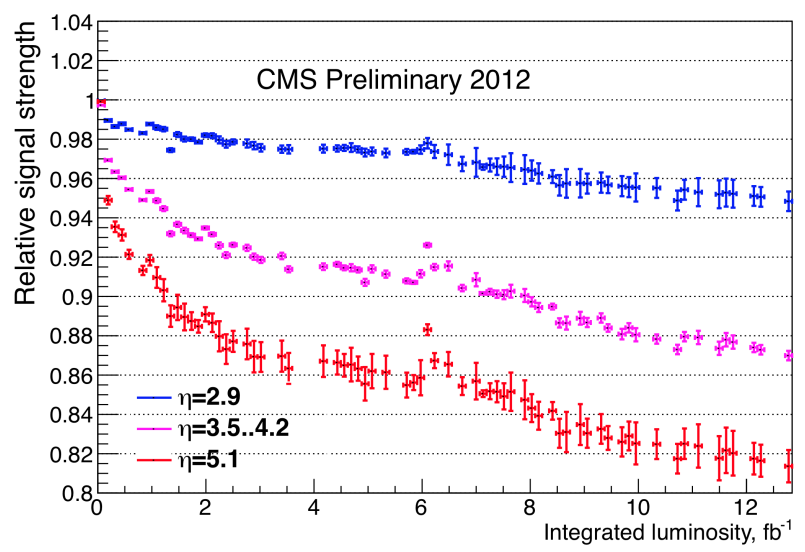

Figure 2: Relative signal loss of the HF PMT's as a function of luminosity since 2010

\subsection{Radiation damage to HF quartz fibers}

To monitor the effect of radiation damage to the quartz fibers in HF, a dedicated system with the HCAL UV laser is used. Laser light is shone into a special radiation damage fiber which runs alongside the regular quartz fibers, the initial reflection and the secondary reflection at the end of the fiber are directed to a PMT, and the ratio is compared as a function of time and luminosity. This quantity, referred to as the Transmission Loss is shown in Figure 3 for 2011 and 2012. The actual signal loss for collision particles is the square root of the transmission loss, as the transmission loss measures the reflected energy loss. Again there is an $\eta$ dependence observed similar to that for the PMT gain loss. The periods of recovery are conincident with LHC technical stops.

\section{HCAL Upgrade Overview}

The Phase 1 upgrade of the CMS HCAL is designed to improve the performance at high luminosities by increasing the depth segmentation (see 4) of the calorimeter and also by improving the ability to reject noise. The HB and HE HPD's will be replaced with Silicon Photomultipliers (SiPM) and the HF single-anode PMT's will be replaced by multi-anode PMT's operating with dual-readout. The electronics used for reading out the signals will also be replaced. 


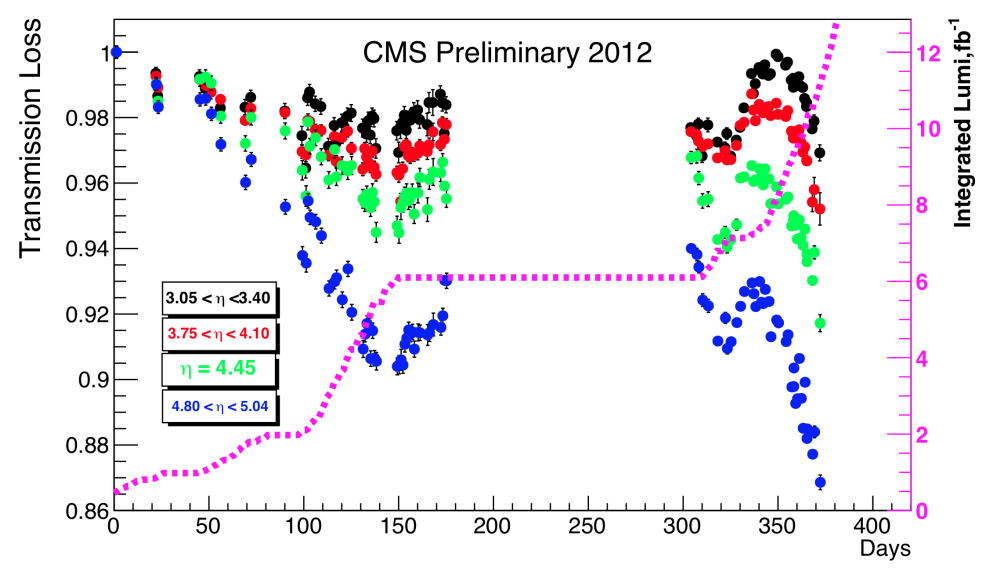

Figure 3: Transmission loss due to radiation damage to the HF quartz fibers

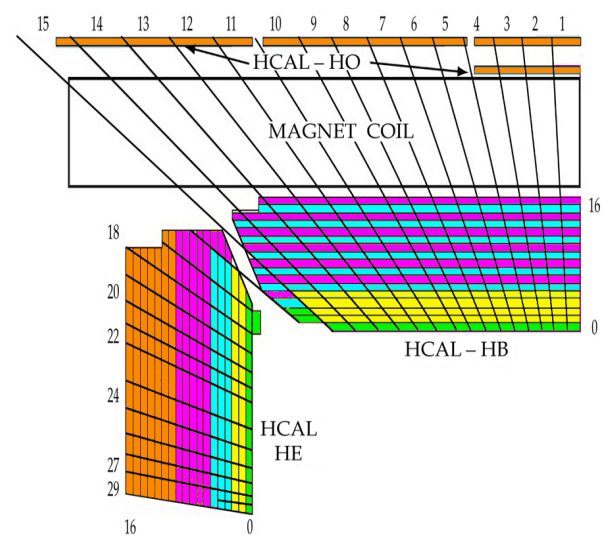

Figure 4: The proposed upgrade to the depth segmentation of HCAL

\subsection{Silicon Photomultipliers in HB, HE, HO}

SiPM's are pixelated avalanche photodiodes and are run in Geiger mode. They are able to provide gains of between $10^{4}$ and $10^{6}$ with an operating voltage of less than $100 \mathrm{~V}$. The devices are compact with a surface area of between $1 \mathrm{~mm}^{2}$ and $9 \mathrm{~mm}^{2}$. It is estimated these devices can improve our signal to noise ratio by a factor of 10 . We have successfully operated prototype SiPM's in 2 sections of the Outer Calorimeter since 2009 to evaluate their performance in real running conditions.

\subsection{HF multi-anode PMTs}

The currently installed single-anode PMTs were observed to suffer from anomalous signals from single which passed through the phototubes. In the intial operation of CMS in 2010, it was more straightforward to identify these in the low luminosity environment, however as the luminosity has increased it has become harder to reject them due to pileup collsions. Furthermore, at 50ns bunch spacing, the current filters to identify these anomalous signals rely on not having collisions in the bunch before or after the primary collision. At $25 \mathrm{~ns}$ bunch-spacing this will no 
longer be possible. Improvements to the HF are thus required to cope with high luminosity and different running conditions. A major piece of this upgrade is the replacement of the Hamamatsu R7525 single anode PMTs with Hamamatsu R7600 multi-anode PMTs. These PMT's have thinner glass windows and metal envelopes. These changes are shown to dramatically reduce the rate of anomalous signals. Furthermore, the ability to use a dual readout system will ensures signals can be compared to identify and reject single-particle anomalous signals. The dual readout system is shown in Figure 5. A block of 24 new PMT's was installed in the HF before the 2012 run, and are recording data well.

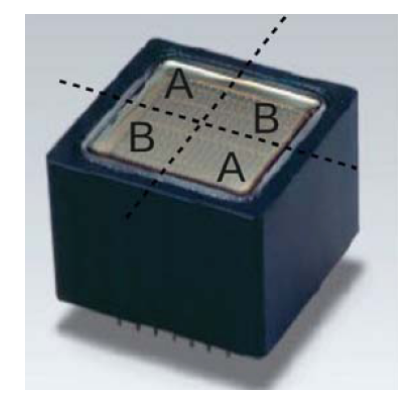

Figure 5: A prototype of the Hamamatsu R7600 multi-anode PMT, to be installed in HF during 2013/2014.

\subsection{Backend electronics}

The upgraded back-end electronics will have the same primary role as the existing back-end system. To meet the requirements of increasing data volume expected with the higher luminosities at the LHC, the backend will be upgraded to use modern FPGA's and the $\mu$ TCA architecture, which is the new standard in the Telecommunications industry. The $\mu$ TCA architecture uses Advanced Mezzanine Cards (AMC) as the standard interface, this new standard is being brought in across CMS. In HCAL a prototype was installed in 2012 and, using optical splitters on the front-end signals, was reading out collision data. An example of the bunch structure from the LHC as read out using the new $\mu$ TCA system is shown in Figure 6.

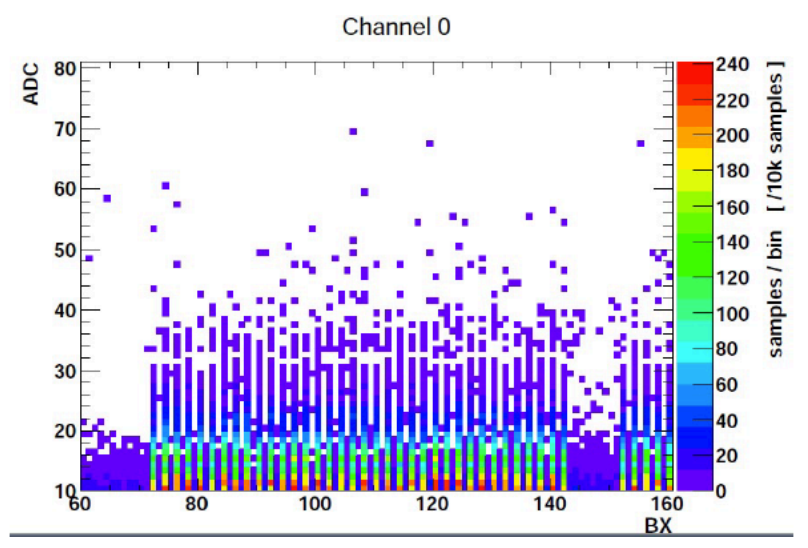

Figure 6: The LHC bunch structure as readout by a prototype $\mu$ TCA crate 


\section{Summary}

The CMS HCAL is am excellent calorimeter, delivering high quality data with high efficiency and more than $99 \%$ of channels live. The calibration system was used to identify potential effects of the photodetectors, which are now understood and accounted for in data-taking. A comprehensive and detailed upgrade plan is underway to help cope with increasing luminosity and high pileup conditions. Many prototypes of future detectors and electronics has been installed and used in real LHC running conditions since the start of LHC operation.

\section{References}

[1] S. Chatrchyan et al. [CMS Collaboration], JINST 3, S08004 (2008).

[2] CERN-LHCC-97-31.

[3] CERN-LHCC-2012-015. 